\title{
Erratum: Multimode Kapitza-Dirac Interferometry with Trapped Cold Atoms [Phys. Rev. Lett. 113, 023003 (2014)]
}

WeiDong Li, Tianchen He, and Augusto Smerzi

(Received 12 March 2016; published 7 April 2016)

DOI: 10.1103/PhysRevLett.116.149901

One of the authors (A. S.) has an additional affiliation: Institute of Theoretical Physics and Department of Physics, Shanxi University, 030006 Taiyuan, China. 